\title{
Erratum
}

\section{"Increased gastro-oesophageal reflux disease in patients with severe COPD." C. Casanova, J.S. Baudet, M. del Valle Velasco, J.M. Martin, A. Aguirre-Jaime, J. Pablo de Torres, B.R. Celli. Eur Respir J 2004; 23: 841-845.}

Unfortunately, one of the co-authors names was presented incorrectly as published. J. Pablo de Torres should read J.P. de Torres. 